\title{
TATU: an Approach for Supporting Tourists with Disabilities to Indoor and Outdoor Navigation using Mobile Devices
}

\author{
Fábio J. Coutinho ${ }^{1}$, Dayvson Sales ${ }^{1}$, Wagner Fontes ${ }^{1}$, Samuel Lucas V. L. \\ Barbosa $^{1}$, Victor Accete ${ }^{1}$, John Davi D. C. Pires ${ }^{1}$, Rebeca de Jesus Brandão ${ }^{1}$, André \\ Luiz de O. Cezário ${ }^{1}$ \\ ${ }^{1}$ Computing Institute - Federal University of Alagoas (UFAL) \\ Av. Lourival Melo Mota, S/N, Tabuleiro do Martins - 57072-970 - Maceió - AL - Brazil \\ \{fabio, dcsales, wsf, slvlb, victoraccete, jddcp, rjb, aloc\}@ic.ufal.br
}

\begin{abstract}
People with disabilities living in Brazil face great difficulties in the tasks of daily life mainly due to the lack of accessibility in public spaces, products and services. In this context, we noticed a lack of a computing tool that embraces both people with visual and hearing impairment. This work presents TATU - a mobile application for both Android and iOS platforms aimed at supporting people with visual or hearing impairment to enjoy Brazilian tourist attractions, including both open-air and indoor spaces. TATU application has an adaptive interface exclusively designed for each of the impairment user profiles, it can work on guided tour mode by indoor navigation using BLE beacons and outdoor navigation using GPS. Our solution was evaluated by three experiments, one of which was carried out with blind volunteers and TATU application obtained satisfactory results for both spacious attractions with the lowest density of collection items and for the smallest spaces.
\end{abstract}

\section{Introduction}

In Brazil, according to the last census, we have about 14 million of people with some form of disability [IBGE 2015]. In addition, from this amount, roughly 1.5 million are blind [Ottaiano et al. 2019] and 2 million have some kind of hearing impairment, of which 344,000 are deaf. Census data also revealed that $20 \%$ of the people with hearing impairment and $16 \%$ of the people with visual impairment reported a strong limitation for performing daily activities [IBGE 2015]. Such fact, mostly, is due to the severe lack of accessible public spaces for disabled people in Brazil.

Since 2018 Federal University of Alagoas (UFAL) has executed a pilot project in Maceió city named TATU (Assistive Technology for Tourism). TATU project intends to promote the accessibility of people with visual or hearing impairment in touristic spaces for culture and leisure by developing a mobile app using BLE beacons and GPS navigation.

Main challenge of the TATU Project is to provide a mobile solution for Android and iOS platforms in order to support visually and hearing impaired people at enjoying tourist attractions with different physical characteristics, including the both open-air tourist attractions (e.g., to visit monuments on Maceió beaches) and indoor environments (e.g., to visit Théo Brandão Museum). 
This work discusses the issues related to the TATU mobile application development and its main complex functionalities such as indoor and outdoor navigation support system and the adaptive interface for different profiles of people with disabilities. Thus, the remainder of this paper is organized as follows. Section 2 discusses some related works. Section 3 describes the TATU approach in detail. Section 4 reports the results of the usability tests and experiments for evaluating our mobile solution. Finally, Section 5 presents the conclusions and future work.

\section{Related Work}

TATU approach relates to several issues studied by researchers from different areas over time such as accessibility, indoor and outdoor location, usability, mobile development, IoT, etc. In this section, the most related works to the TATU approach are discussed and organized in the subsections 2.1 and 2.2.

\subsection{Usability and Accessibility}

People with disabilities living in Brazil face great difficulties in the tasks of daily life mainly due to the lack of accessibility in public spaces, products and services. In this context, when we consider groups of blind and deaf users, we realize the need to create user profiles with completely different approaches as can be verified in WCAG (Web Content Accessibility Guidelines) [W3C 2018] - a W3C initiative to provide accessibility to Web applications. Usability designed for blind people, in general, involves the use of features such as screen readers, audio messages for guidance and vibration [Morris et al. 2018, Ramadhan 2018]. For deaf users, the usability should prioritize features such as light, shadow, color contrast and visual alerts to replace sound minimizing the use of texts, videos or animations with sign language, captioning and sign language translators [Coutinho et al. 2011, Nathan et al. 2018].

To the best of our knowledge, we did not find any work that has developed a mobile application with usability designed specifically for the blind and also for the deaf. All works only focus on a specific group of people with disabilities. Ahmetovic et al. NavCog [Ahmetovic et al. 2016, Sato et al. 2017] implemented an app for indoor navigation aimed specifically to help people with visual impairments. The results presented by the authors are positive for indoor location. Unlike TATU which includes deaf users and an approach to outdoor navigation support, $\mathrm{NavCog}$ is restricted to blind and low vision users. In addition, NavCog is a proprietary solution used exclusively for the iOS platform.

\subsection{Navigation and Localization}

Exploring places that we are not familiar with can be a problem for most people, however, when we regard the mobility of people with disabilities, this challenge becomes even greater. Thus, several research works have studied alternatives to help the mobility of people with visual impairments, mainly, in spaces visited for the first time. Initially, some works explored the technologies Ultra-wideband [Gezici et al. 2005], Ultrasound [Priyantha et al. 2000] and Infrared [Aitenbichler and Muhlhauser 2003]. More recently, several works have improved indoor location accuracy using Wi-Fi from the inclusion of multiple access points and/or additional hardware/software components [Kotaru et al. 2015, Xiong et al. 2015]. 
RFID is another technology that has been explored by several indoor localization works. A representative example was proposed by Guerrero et al. [Guerrero et al. 2012] who developed an RFID-based system where the blind uses a smartphone along with an augmented white cane containing infrared emitters. In addition, the installation of two Wiimote (game controller with integrated infrared camera) is also required to guide the user through the planned path. Despite demonstrating a good accuracy, the work demands hardware that can be costly when applied in different spaces of the attraction. Due to the financial factor, its use in tourist attractions of the Brazilian cities becomes untenable.

Recently, some works have presented good results using Bluetooth Low Energy (BLE) beacons for indoor navigation as discussed in [Jeon et al. 2018]. Singh et al. [Singh et al. 2018] developed an indoor navigation software to smartphones and tablets using beacons and Dijkstra's algorithm to calculate the available shortest path from the source to the desired destination. Ahmetovic et al. developed a turn-by-turn navigation system for blind people using BLE beacons with an approach based on the K-nearest neighbor (KNN) algorithm [Ahmetovic et al. 2016].

\section{TATU Approach}

TATU approach uses an indoor and outdoor navigation support mobile system designed to allow blind and deaf visitors to enjoy tourist attractions autonomously and independently, i.e., without the need for external mediation (tour guide). Thus, TATU system has two kinds of navigation support that are activated according to the type of attraction visited: indoor navigation mode using BLE beacons and outdoor navigation mode for open-air tourist attraction using GPS.

Unlike the other solutions, TATU App navigation support system does not have the ultimate goal of taking the user from a specific point to another specific destination point. Instead, the application focus on helping the user to find accessible items from a collection (e.g., artwork, monument, etc.) as he/she freely explores the environment, in case of a blind user, using his/her cane, tactile floor, guide dog and other possibles resources along with the estimated location information provided by TATU navigation system.

This difference is mainly due to the context of the problem addressed in TATU Project, where the user experiences the visit to a tourist attraction which is distinguished, for example, from proposals where the user, in travelling, transits in big airports or train stations. Figure 1 presents an overview of TATU App working on indoor navigation support mode in a museum.

\subsection{Implementation}

TATU application was developed using the Ionic Framework [Ionic 2021], which allows cross-platform development with a single code using web technologies (HTML, JavaScript and CSS). Applications developed with Ionic are rendered using the WebView provided by the mobile operating system. Using Apache Cordova [Cordova 2021], Ionic can provide access to the native capabilities of operating systems (such as camera, microphone and GPS).

During prototype testing, it was verified that some Ionic accessibility features are limited. For instance, WebView does not support some ARIA tags on iOS and components 


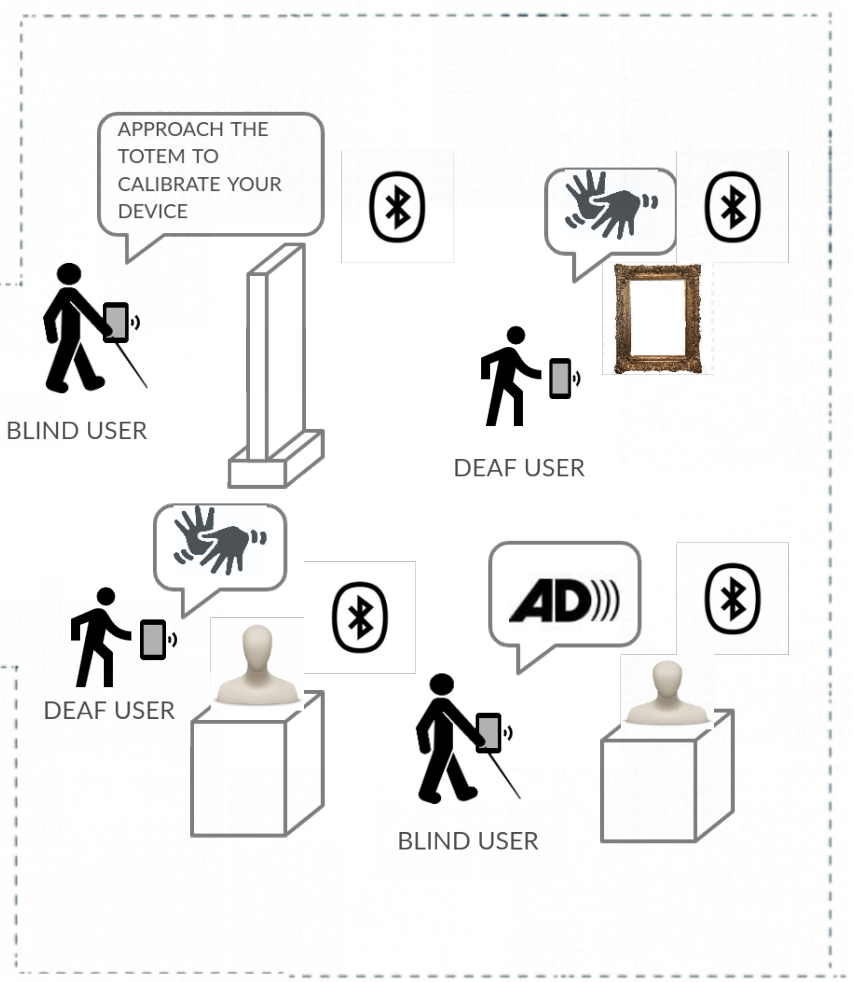

Figure 1. TATU App overview working on indoor navigation support mode.

such as ListView, Slider, and AlertBox, are not properly adapted for screen readers. In order to address these issues, we have developed alternative versions of these components adapted for screen readers (especially Talkback and VoiceOver). Thus, the user can easily use components that were not originally accessible.

\subsection{Indoor Navigation}

The purpose of the indoor navigation support system in TATU application is to assist the user in exploring the accessible works inside buildings through the use of Bluetooth Low Energy (BLE) beacons. The use of beacons allows inferring the user's approximate distance to the artwork by the Received Signal Strength Indication (RSSI) without the need to track the user all the time in a region, but only at specific predetermined points. Therefore, beacons are used to find accessible artworks and to assist the locomotion of blind users by sending information about the internal space (e.g., You are about 5 meters from the access stairway to the second floor; bathroom is at the end of this corridor on the left, etc.). Each beacon is attached to an accessible artwork in order to allow the application to identify it (by a unique identifier number) for retrieving its information. A single beacon is exclusively used in the calibration process for each tourist attraction, as described in the following explanation.

Since RSSI measurements strongly differ on smartphones from different manufacturers even under identical circumstances [Ma et al. 2017], we developed a calibration mechanism to the user devices uniformly work. Using this mechanism is possible to establish the desirable minimum distance to the work be detected by the TATU application in the attraction. Thus, the calibration can be described as a procedure to define a proper distance for the detection of the beacon in each visited attraction. 
Upon entering the attraction the user must approach to a totem previously positioned at a specific distance from a beacon reserved for this procedure denoted calibration beacon. This process is implemented from a set of RSSI values previously collected in order to determine a threshold based on the mode (most frequent value) of the approximate distances.

1. Initially, a sample of RSSI values is collected for a maximum period of 10 seconds.

2. From the RSSI values, a vector is created with approximate values for the distance between the user (totem) and the calibration beacon.

3. Calculates the mode of the approximate distances in the vector and apply a method for meeting outliers.

4. Check if the mode obtained belongs to the detected outliers, if it belongs back to step 1 for collecting a new sample of RSSI values, otherwise, the mode value is saved to be used as the distance threshold for detecting works in the attraction.

Figure 2 displays graphs with distance vector values obtained during calibration. The $y$-axis shows the approximate distance values and $\mathrm{x}$-axis indicates the index corresponding to each sample unit. The graph allows us to verify that the RSSI values collected vary even if the reading position (smartphone's position in relation to the calibration beacon) does not change.

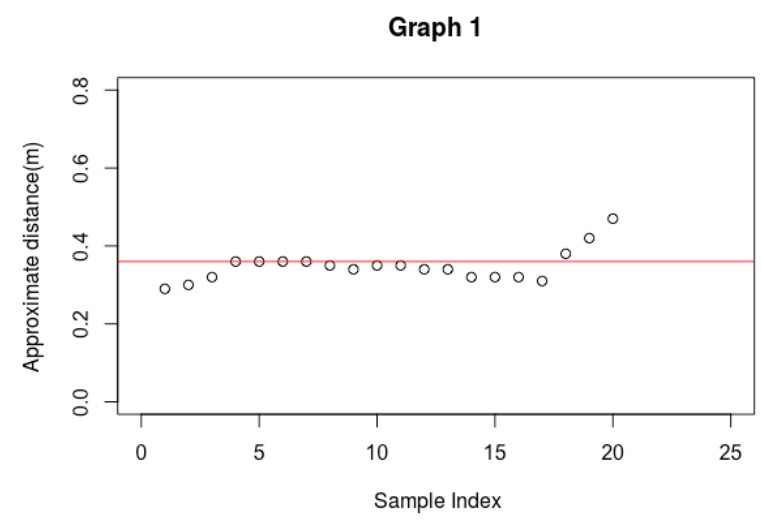

(a) First calibration test

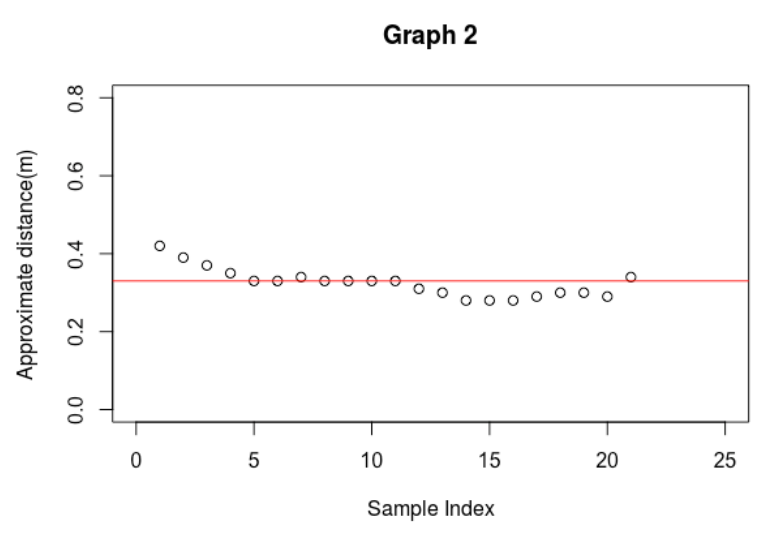

(b) Second calibration test

Figure 2. Graphs with approximate distances obtained from RSSI values collected during the calibration process.

After calibrating his/her smartphone, the user will be ready to start the navigation mode. In this mode, the application performs a search for beacons that are close to the user, these beacons are associated with artworks (painting, statue, etc.). Thus, when a beacon is detected, the distances between the smartphone and the target beacon are constantly calculated. If the user is close enough to the beacon, i.e., his/her distance is within the threshold established in the calibration, a screen is displayed providing the accessible content related to the work associated with the detected beacon (e.g., audio description). The user also can choose to access this content later and continue searching for other beacons as he/she moves around the attraction, this process will take place until the user leaves the navigation mode. 


\subsection{Outdoor Navigation}

In outdoor navigation, TATU application uses GPS technology instead of beacons. The main reason for declining the use of beacons in open-air environments was the difficulty in ensuring the integrity of the devices due to the constant occurrence of acts of vandalism in works exposed in open-air places in Brazil. Another motive is that works exposed in open-air places usually are not very close to each other, requiring a lower degree of accuracy in detection. In summary, Haversine formula, a known method to calculate the distance between two points in a sphere, is used to implement the calculation of the distance between a work to be detected and the current position user.

\subsection{Usability}

People with disabilities face several problems when visiting tourist attractions in Brazil. For most destinations, it is not possible to ensure that this public can experience a visit on equal terms with the other people. In this sense, TATU Project seeks to mitigate this adversity by developing a mobile application to support the visitation of the blind and deaf people. To achieve this goal, the usability plays a key role in the implementation of an effective solution that promotes the interest of the target audience.

User experience design in the TATU app development had the participation of two blind collaborators, three deaf and one Portuguese-Libras translator. This team worked from the conception of the wireframes to the final version, collaborating with three prototypes during the development phase. In addition, a survey conducted with blind people was carried out in order to know the usability characteristics considered relevant in mobile applications and some apps for disabled people were evaluated by the UX team.

When the TATU application is started for the first time, the user is asked which kind of accessibility profile he or she intends to choose: (i) audio descriptions for visual impairment people; (ii) Libras sign language for deaf people and (iii) no accessibility, as shown in Figure 3. According to the provided answer, the application will run a distinct interface to ensure the best available experience for each audience as presented in Figure 4.

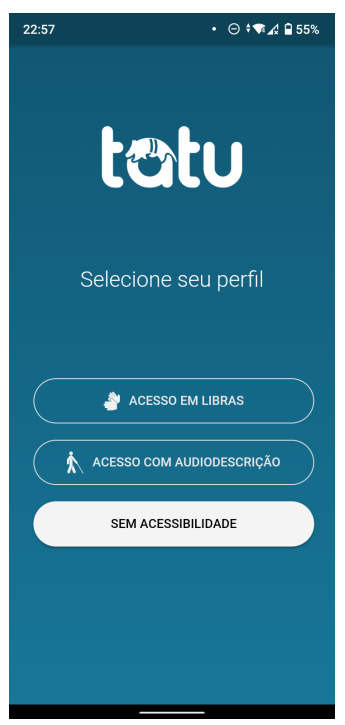

Figure 3. Accessibility profile selection screen 


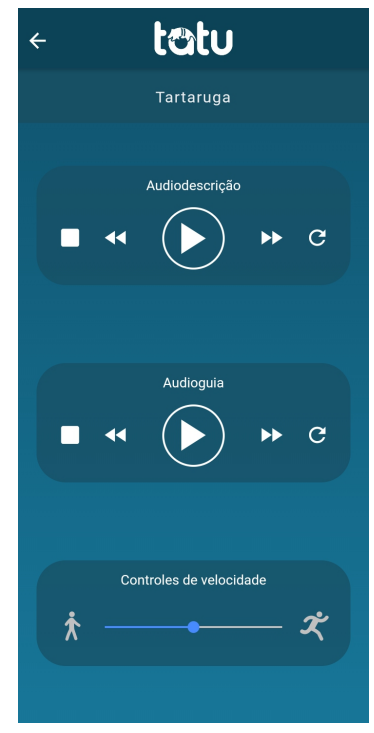

(a) For blind users

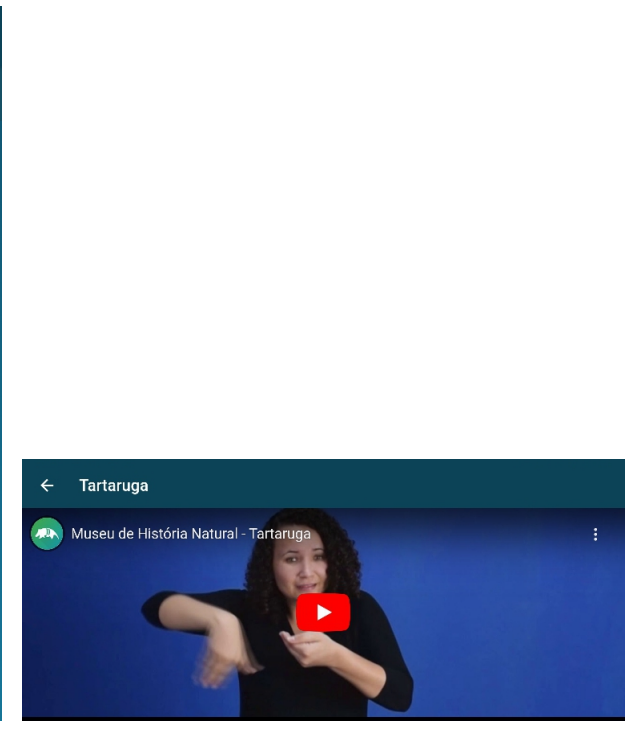

(b) For deaf users

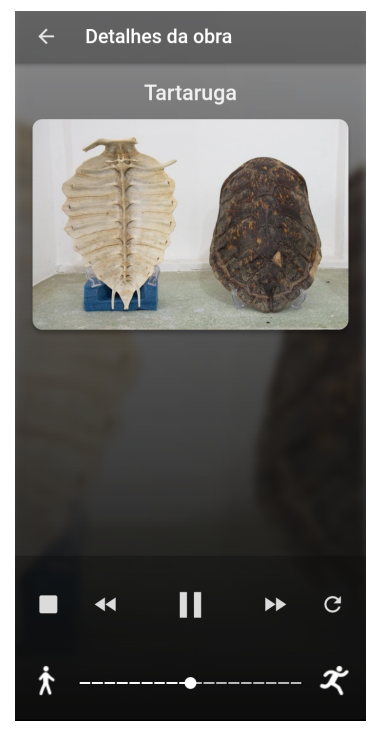

(c) For non-disabled users

Figure 4. Content screens for distinct user profiles.

Based on the feedback received from blind respondents, TATU usability was designed to minimize user interaction with the app and facilitate the interaction through the screen reader used in Android (TalkBack) and iOS (VoiceOver). In addition, it was identified the need to have a speech synthesizer (text-to-speech - TTS) to work together with the screen reader. TTS offers the possibility of emitting sound feedback to the blind users about the state of the app reducing the interaction requirement. Usability for the deaf user profile was also guided by the participation of hearing-impaired collaborators. Among the recommendations adopted by the development team are: avoid the textual information by replacing, whenever possible, with images of similar meaning or sign language videos; preference for the use of short texts; and prioritization of colloquial language.

In general, TATU application usability sought to minimize user interactions as he or she appreciates the visited attraction. Other relevant aspects related to the user experience are described as follows.

1. The layout of the application pages (text, image and icon positioning) was designed according to the Nielsen heuristics.

2. The identification of all visual elements (graphics and texts) followed the WAIARIA guidelines [W3C 2019].

3. Removal of non-essential elements to meet the usability according to the user interface profile.

4. Information related to the collection of attractions was researched by a specialized team in order to produce the appropriate accessibility resources: audio descriptions and audio guides for the blind; sign language videos for the deaf and audio guides for non-disabled non-disabled users.

\section{Evaluation}

Considering the interdisciplinary nature of the knowledge addressed in TATU project, its execution had the participation of different research groups from the Federal University 


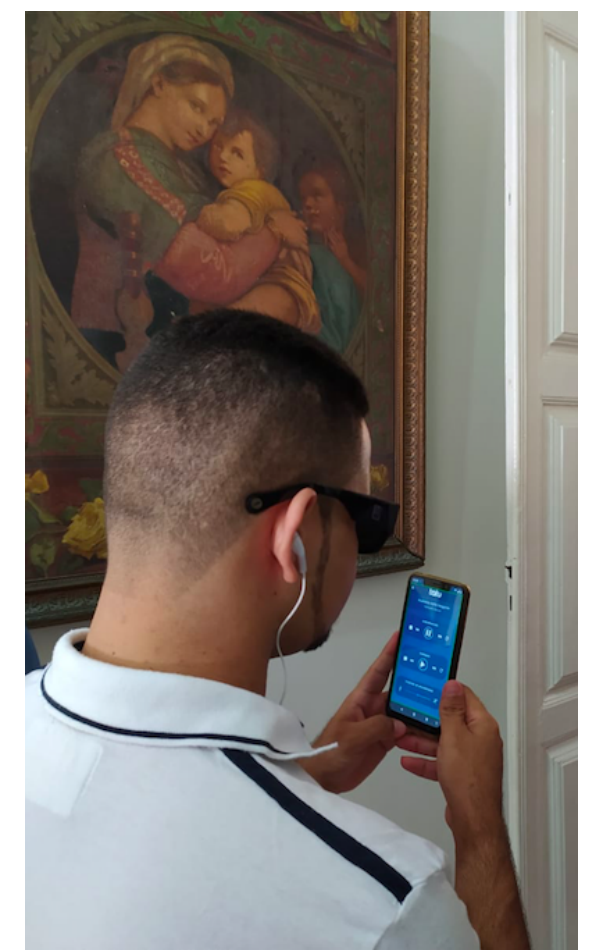

Figure 5. A blind volunteer testing TATU app in visiting to the MUPA.

of Alagoas who worked on distinct fronts related to the application development. The evaluation process of the TATU proposal occurred in two ways: internal - through the validation of the prototypes by blind and deaf collaborators of the project team; and external - based on experiments performed in tourist attractions having the participation of volunteers with disabilities who do not belong to TATU project team.

The evaluation of the implemented prototypes was part of the evolution of the iterative development cycle applied in the TATU project execution. In this sense, some sessions were held with the participation of up to two collaborators with disabilities for the analysis of the prototype. Evaluation sessions took place as described below. Initially, development team prepared the environment by positioning some beacons used in the test. Next, development team gave basic instructions to the testers explaining the procedure. Finally, a report was produced based on the considerations made by the testers. The reports were used by the development team to satisfy the demands of the testers by improving the implementation of the application.

Furthermore, an experiment was performed to evaluate the use of the TATU application in indoor space with six volunteers whose visual acuity category is NPL in both eyes - total blindness. The main objective of this experiment was to evaluate the TATU application taking into account usability aspects in the blind user profile and the effectiveness of indoor navigation support. Initially, the volunteers were instructed on how to download the app and calibrate it ahead of filling out one form before the visit and another after. The form aimed to collect information related to the volunteer's personal data for a better understanding of the tester profile. Among the information collected are: age group, gender, frequency of visits to museums and familiarity with using of assistive technologies in museums. 
For this experiment, 21 beacons of the Smart Beacon SB16-2 model made by Kontakt.io were used and the volunteers successfully calibrated their smartphones (4 running on Android and 2 on iOS) between $0.9 \mathrm{~m}$ and $1.5 \mathrm{~m}$. Each volunteer was free to interact with the TATU app on indoor navigation mode and explore the environment using the smartphone and its cane as shown in the Figure 5.

Finally, volunteers filled out a second form that aimed to assess their degree of satisfaction with the visit assisted by the application and whether the application met expectations regarding usability and accessibility. The following nine statements were presented to the volunteers, who anonymously expressed themselves after the visit.

1. The application is easy to use.

2. The application is accessible.

3. The application has improved your museum experience.

4. Using the application, you would visit tourist sites more often.

5. You would use the app to go to tourist places with someone.

6. Audio descriptions were satisfactory and fulfilled their purpose.

7. You considered the amount of accessible works sufficient.

8. Audio guides improved the experience of the visit.

9. You did not miss a tour guide during the visit.

The degree of agreement of the volunteers was measured using the Likert scale and the answers are presented in the graph shown in Figure 6. Level of agreement attributed to the statements 1,2 and 6 confirms that TATU mobile application met the expectations of usability and accessibility, which represent its main objectives. The answers to the statement 9 indicate a possible claim by some volunteers for the presence of a tour guide to assist in visiting. However, the cost to provide a tour guide trained in providing audio descriptions and fluent in Libras sign language would be too high for the financial situation of most Brazilian attractions.

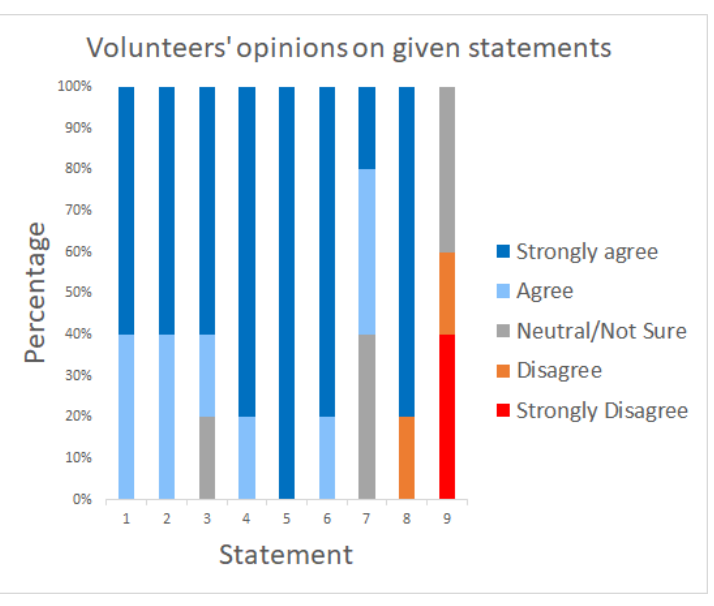

Figure 6. Answers given anonymously by opinion form.

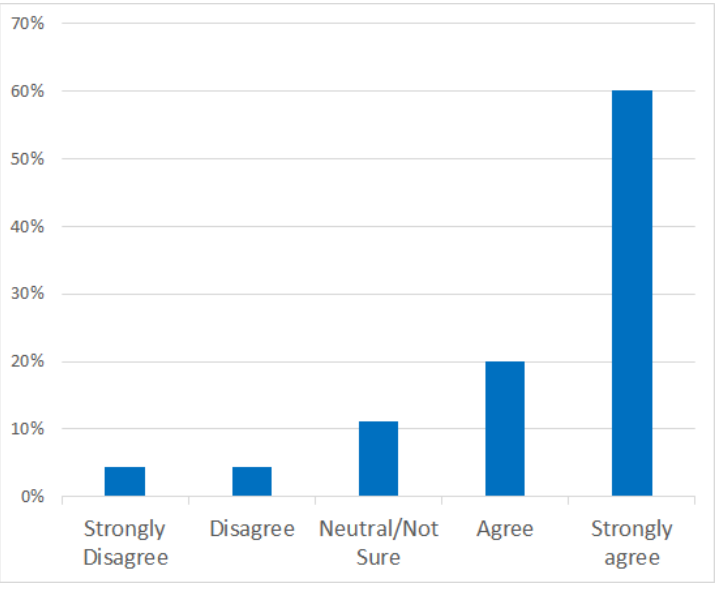

Figure 7. An aggregated overview of all provided responses.

Figure 7 displays a graph that represents an aggregated overview of all responses provided by volunteers and calculated by accumulating the number of responses assigned to each of the five Likert scale options. Since all nine statements address a positive value to the TATU solution, the volunteers' high degree of agreement with the statements reveals the good acceptance of our solution as can be seen in Figure 7. 


\section{Conclusion and Future Work}

In Brazil, people with disabilities face a series of difficulties in their daily lives, including visiting tourist spaces, such as museums. This work presented TATU application developed to help the user with visual or hearing impairment to find accessible information as it explores the tourist attraction. TATU app provides the guided tour functionality that allows indoor navigation through BLE beacons and outdoor navigation using GPS. It has an adaptive interface which is elaborated from different usability designs that aim to provide an adequate experience to the needs of each user profile - deaf, blind and non-disabled.

The experiments carried out for the evaluation of the TATU solution showed that the visit aided by the app obtained satisfactory results for both spacious attractions with the lowest density of collection items and for the smallest spaces with the highest density as detailed in the section 4 . This fact reveals the success of our proposal in order to implement a generic development solution to be applied in different tourist spaces serving blind, deaf and non-disabled users.

In future, we intend to expand the TATU solution to include new user profiles with disabilities. In addition, it is also aimed to implement machine learning techniques on the data collected during the device calibration and the guided tour in order to improve the indoor navigation support and automate the calibration process in future versions.

\section{Acknowledgement}

We appreciate the valuable contribution of researchers, museologists, designers and the audio guide, audio description and sign language video production team. This work was financed in part by the National Council for Scientific and Technological Development (CNPq), Foundation for Research Support of Alagoas (Fapeal), Federal University of Alagoas (UFAL) and Ministry of Tourism.

\section{References}

Ahmetovic, D. et al. (2016). Navcog: A navigational cognitive assistant for the blind. In Proceedings of the 18th International Conference on Human-Computer Interaction with Mobile Devices and Services (MobileHCI'16), pages 90 - 99.

Aitenbichler, E. and Muhlhauser, M. (2003). An IR local positioning system for smart items and devices. In Proceedings of the 23rd International Conference on Distributed Computing Systems Workshops (ICDCSW 2003), pages 334-339.

Cordova, A. (2021). What is Apache Cordova? https: //ionic.io/resources/ articles/what-is-apache-cordova. [Accessed: 2021-06-05].

Coutinho, F. et al. (2011). An analysis of information conveyed through audio in an fps game and its impact on deaf players experience. In Proceedings of the X Brazilian Symposium on Computer Games and Digital Entertainment (SBGames 2011), pages 53-62.

Gezici, S. et al. (2005). Localization via Ultra-Wideband Radios: A Look at Positioning Aspects for Future Sensor Networks. IEEE Signal Processing Magazine, 22:70-84.

Guerrero, L. et al. (2012). An Indoor Navigation System for the Visually Impaired. Sensors, 12(6):8236-8258. 
IBGE (2015). Pesquisa nacional de saúde: 2013: ciclos de vida: Brasil e grandes regiões. IBGE. (In Portuguese).

Ionic (2021). Ionic framework. https : / / ionicframework . com. [Accessed: 202106-05].

Jeon, K. E. et al. (2018). BLE Beacons for Internet of Things Applications: Survey, Challenges and Opportunities. IEEE Internet of Things Journal, 5(2):811-828.

Kotaru, M. et al. (2015). SpotFi: Decimeter Level Localization Using WiFi. In Proceedings of the 2015 ACM Conference on Special Interest Group on Data Communication (SIGCOMM'15), page 269-282.

Ma, Z., Poslad, S., Bigham, J., Zhang, X., and Men, L. (2017). A BLE RSSI ranking based indoor positioning system for generic smartphones. In 2017 Wireless Telecommunications Symposium (WTS), pages 1-8.

Morris, M. et al. (2018). Rich Representations of Visual Content for Screen Reader Users. In Proceedings of the 2018 CHI Conference on Human Factors in Computing Systems, page $1-11$.

Nathan, S. et al. (2018). Usability evaluation of DEAF mobile application interface: A systematic review. Journal of Engineering and Applied Sciences, 13(2):291-297.

Ottaiano, J. et al. (2019). As Condições de Saúde Ocular no Brasil 2019. Conselho Brasileiro de Oftalmologia. (In Portuguese).

Priyantha, N. B., Chakraborty, A., and Balakrishnan, H. (2000). The Cricket LocationSupport System. In Proceedings of the 6th Annual International Conference on Mobile Computing and Networking (MobiCom '00), page 32-43.

Ramadhan, A. J. (2018). Wearable Smart System for Visually Impaired People. Sensors, 18(3, 843):1-13.

Sato, D. et al. (2017). NavCog3: An Evaluation of a Smartphone-Based Blind Indoor Navigation Assistant with Semantic Features in a Large-Scale Environment. In Proceedings of the 19th International ACM SIGACCESS Conference on Computers and Accessibility (ASSETS '17), page 270-279.

Singh, A. et al. (2018). Indoor navigation system using bluetooth low energy beacons. In Proceedings of the Fourth International Conference on Computing Communication Control and Automation (ICCUBEA 2018), pages 1-5.

W3C (2018). Web Content Accessibility Guidelines (WCAG) 2.1. https : / / www . w3 . org/TR/WCAG21. [Accessed: 2021-03-12].

W3C (2019). WAI-ARIA Authoring Practices 1.1. https://www.w3.org/TR/ wai-aria-practices-1.1/. [Accessed: 2021-03-12].

Xiong, J. et al. (2015). ToneTrack: Leveraging Frequency-Agile Radios for Time-Based Indoor Wireless Localization. In Proceedings of the 21st Annual International Conference on Mobile Computing and Networking (MobiCom '15), page 537-549. 